\title{
MEASUREMENT ISSUES OF RADIO FREQUENCY INTEGRATED CIRCUITS WITH DIGITAL CONTROL AT RADIATION TESTING
}

\author{
Konstantin Amburkin", George Chukov, Vadim Elesin, Galina Nazarova, Nikolay Usachev \\ National Research Nuclear University MEPhI (Moscow Engineering Physics Institute), 115409, \\ Moscow, Russia
}

\begin{abstract}
The results of the development of a portable logical generator/analyzer to be used in an automated hardware and software measurement system for automation of research and testing of TID hardness of digitally controlled RF ICs, are provided. The device has been tested in research of precision parameters of integrated multi-bit RF vector phase shifters.
\end{abstract}

\section{Introduction}

In electronic components development today, there is an efficiency-driven trend to combine the functions of an RF transceiver path, digital control and signal processing on a single chip $(\mathrm{SoC})$ or in a single package (SiP). Current and prospective RF transceiver ICs are complex analog-digital functional blocks [1-5]. A comprehensive radiation testing of such RF ICs is possible only if the functions of automated logic control and management have been built in a RF hardware/software measurement system [6].

An example of such an analog-digital block for transceiver modules of RF active phased arrays, is a monolithic IC designed for phase control (6-bit) and amplitude control (5-bit) phase shifter/attenuator (PSA IC) [7, 8]. Phase and amplitude control is implemented via a parallel or serial digital code. Precision parameters of PSA ICs are determined from results of measuring the frequency dependency of S-parameters in each of the 2048 logical states of the IC. If the parallel control code is specified using mechanical switches (jumpers) and then saved as an S-parameter file, this may require several hours of continuous engineer work, which is unacceptable under the radiation testing [9-11].

To test such ICs, a logical signal generator and logical analyzer had to be introduced into the RF hardware/software measurement system [6]. Commercially available universal logical analyzers cost dozens of thousands of dollars, and their considerable size obstructs their use in radiation testing of RF electronic components in radiation facilities [12-15].

This necessitates developing of an inexpensive portable logical analyzer/generator (PLAN-16M) integrated with a RF hardware/software measurement system and adapted for radiation testing.

Corresponding author: kmamb@spels.ru 


\section{PLAN-16M device description}

PLAN-16M has two eight-bit bidirectional ports with variable supply voltage; the device is controlled from a personal computer (PC) via a USB interface.

The functional block diagram of PLAN-16M is shown in Figure 1; it consists of a microcontroller (MCU) enabling connectivity with a PC and managing other IC nodes, bidirectional buffers and a programmable power supply. Two independent external ports allow the device to operate both as a logical analyzer and logical signal generator.

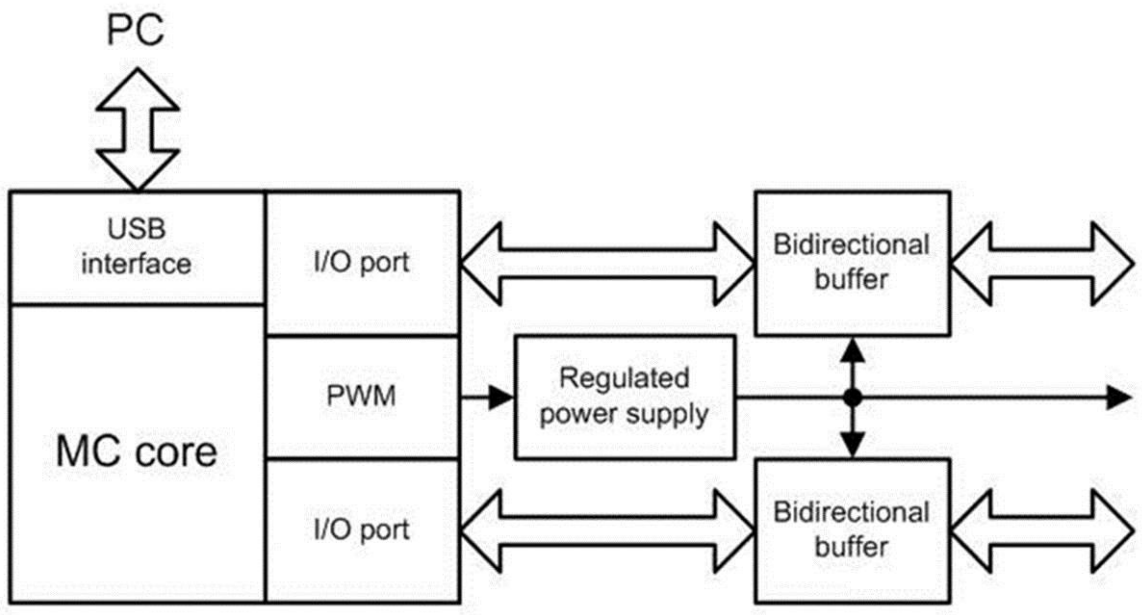

Figure 1. Functional block diagram of PLAN-16M.

The core of the device is the AT90USB162 MCU (Atmel) [16], selected based on the following criteria: availability of a hardware USB interface, required number of $I / O$ pins, simple architecture, high availability and low cost. To implement bidirectional ports with variable levels of logic signals, bidirectional buffer ICs 74LVX3245 have been used [17]. The device is fully powered via a USB port. Buffer ICs can be powered both via a built-in programmable source and an external source, to expand the range of levels of logical signals supported. The built-in programmable power supply has been implemented based on the MCU's hardware PWM module and an integrating filter based on the AD826AN operational amplifier (Analog Devices) [18]. Also, the RF IC tested can be powered directly by the built-in programmable PLAN-16M internal power supply. The exterior PLAN-16M is shown in Figure 2. Key features of PLAN-16M are shown in Table 1.

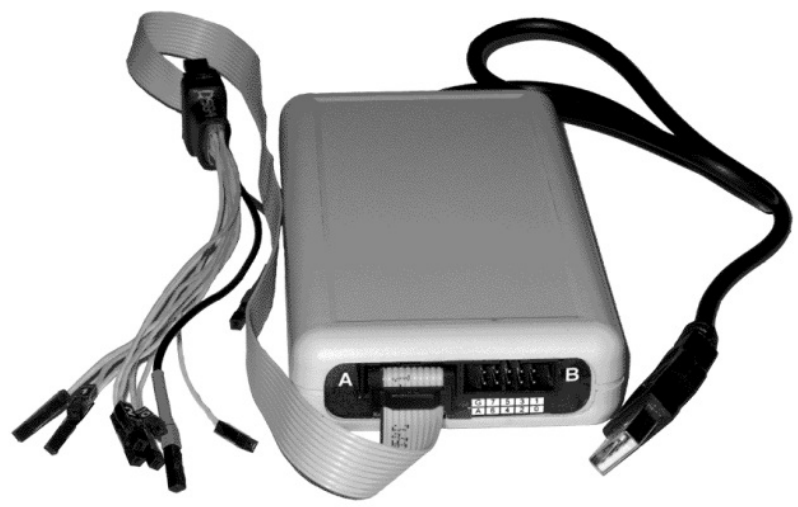

Figure 2. Exterior of PLAN-16M. 
Table 1. Key features of PLAN-16M.

\begin{tabular}{|l|c|}
\hline \multicolumn{1}{|c|}{ Feature } & Value \\
\hline Number and bit of I/O ports & $2 \times 8$ \\
\hline Supply voltage, V & 5 \\
\hline Range of logical signals I/O ports levels, V & $1.5 \ldots 5$ \\
\hline Parallel data setup time code, ms & $<2$ \\
\hline Supply Current, mA & 60 \\
\hline Package size, mm & $134 \times 68 \times 24$ \\
\hline
\end{tabular}

When connected to a PC, PLAN-16M is recognized by the system as a standard USB HID-class device with no additional drivers needed to run it. To communicate with the device, a standard library of I/O functions provided by the MCU AT90USB162 manufacturer, is used [19]. To optimize subsequent development of control programs, a specialized library of higher-level functions has been developed. Figure 3 shows a window of the vector circuit analyzer with the program allowing to test PSA ICs both with parallel and serial control.

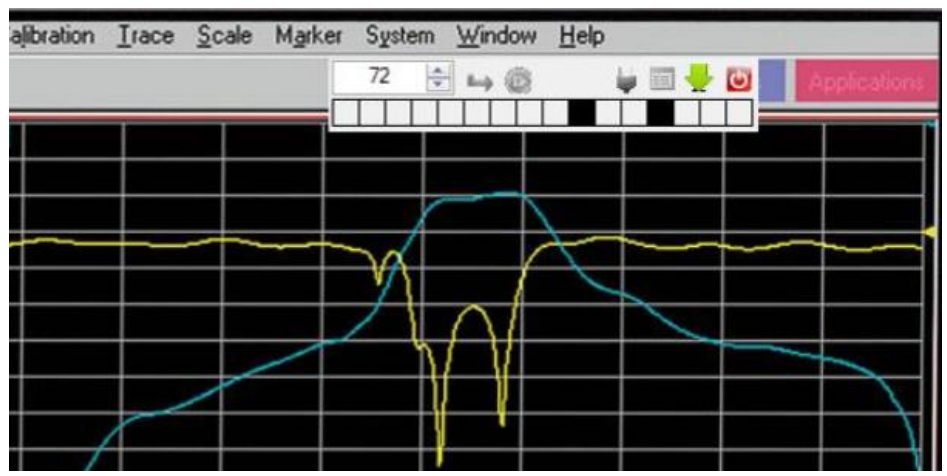

Figure 3. A window of the vector network analyzer with the PLAN 16M control program.

\section{Use of the device IN test of parameters of RF phase shifter and attenuator ICs}

Testing of multi-bit RF PSA ICs with parallel or serial control has some specifics. First of all, measuring and processing of the phase shifter parameters is a resource intensive process, and also RF test fixture is quite complex.

PSA parameters are classified into primary parameters, i.e. those measured directly, and secondary parameters, which are calculated based on the primary parameters $[7,8]$. The primary parameters are S-parameters, the dynamic range parameters (noise Figure and input $1 \mathrm{~dB}$ compression point). Secondary parameters are the integral or precision parameters including root mean square (RMS) for the phase error $\left(\mathrm{RMS}_{\varphi}\right)$ and for the amplitude error $\left(\mathrm{RMS}_{\mathrm{A}}\right)$.

Using the device developed, the test routine for multi-bit RF PSA ICs has been automated; the core of this process constitutes measuring of primary parameters and subsequent calculation of secondary PSA ICs parameters. The test routine based on a RF hardware/software measurement system integrated with RF CAD and the universal RF test fixture characterized in a frequency range of $12.5 \mathrm{GHz}$ [20].

The diagram of 2048 states of the amplitude-phase characteristics of the monolithic RF PSA IC including a 6-bit vector phase shifter and a 5-bit attenuator designed by "Progress MRI" with SiGe BiCMOS process [8], is shown in Figure 4. The diagram shows that testing of such ICs, particularly for radiation hardness, is almost non-practicable without 
the automation of measurement processes (2048 matrices of S-parameters) and mathematical processing of measurement results in real-time (i.e., calculation of $\mathrm{RMS}_{\varphi}$ and $\mathrm{RMS}_{\mathrm{A}}$ used as integral criteria parameters).

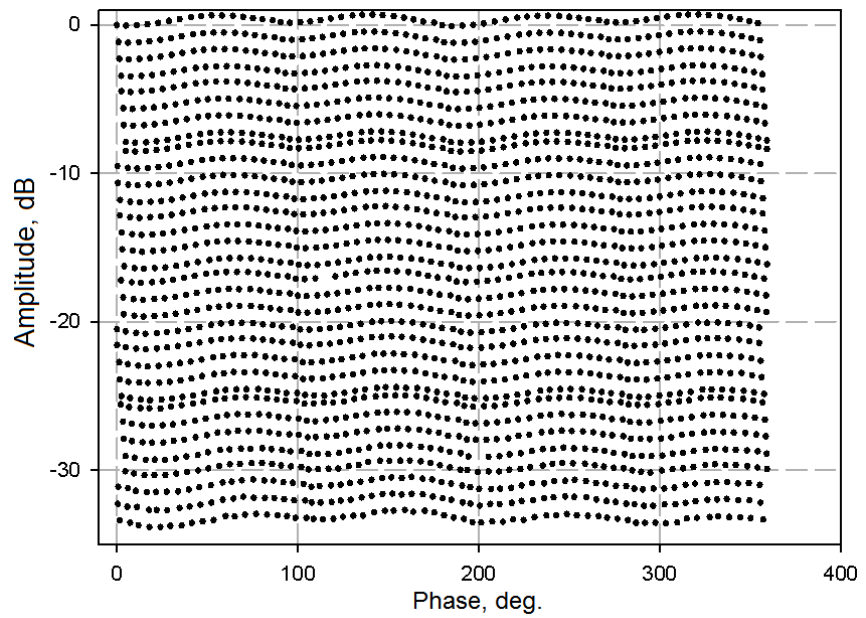

Figure 4. Results of measuring the amplitude-phase characteristics of the PSA IC at a frequency of 5 GHz with PLAN-16M.

\section{Optimazation of precision parameters of vector phase shifters with redundant coding}

Using PLAN-16M, precision parameters of RF vector phase shifters ICs designed by "Progress MRI" with SiGe BiCMOS process, have been measured [8].

RF vector phase shifters ICs are based on combining of two quadrature signals of a variable amplitude. The input signal is split into quadrature components $I$ and $Q$ passed through stepped attenuators and then combined back. The degree of attenuation in attenuators determines the amplitude of signals $I$ and $Q$, and hence the amplitude and phase of the output signal $[8,21]$. For N-bit attenuators, the number of phase shifter states is $2^{2 \times N}$; from this set, the basic states are selected, i.e. those which enable phase adjustment at a given step without the amplitude change. Such states are usually selected at the design stage; however the manufacturing tolerance may result in a substantial degradation of phase shifter precision parameters. A way to improve the phase shifter precision parameters is to select the main states after manufacturing.

The procedure used to optimize the phase shifter precision parameters by selection of the initial main operating states based on testing results, consists of two main stages: at the first stage, complex transmission coefficients (S21) are measured in all states of the phase shifter; at the second stage, the initial states are checked and selected based on the given criteria.

The RF hardware/software measurement system [6] has been used at the first stage; to automatically run a complete set of measurements needed for all the phase shifter states, PLAN-16M has been used. Block diagram of the measuring set up is shown in Figure 5. The measurement results have been saved as a file containing complex matrices of Sparameters in the frequency range relevant to each state. 
At the second stage, the specialized software searches the main states based on the given criteria which may include $\mathrm{RMS}_{\varphi}$ or $\mathrm{RMS}_{\mathrm{A}}$, amplitude modulation $(\Delta \mathrm{A})$, average insertion loss $\left(A_{a v}\right)$

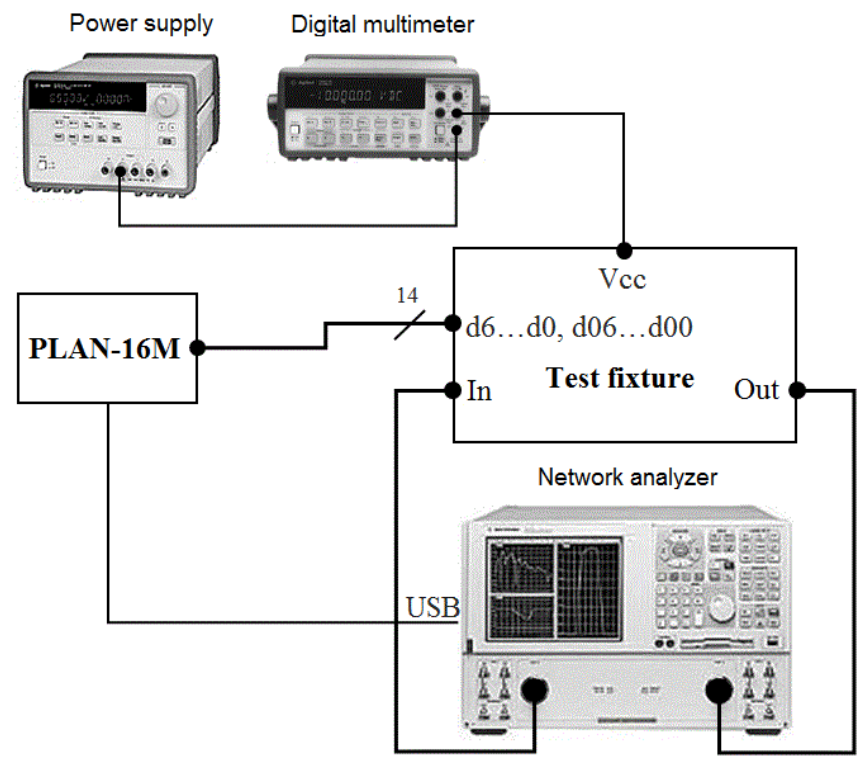

Figure 5. Block diagram of a measuring set up for multi-bit vector phase shifter ICs.

Experimental results obtained for a test 6-bit phase shifter IC operating at $3 \mathrm{GHz}$ and based on 7-bit attenuators (more than 16,000 states total), in the main 64 states according to the specification, have showed unacceptable degradation of its precision parameters (see Table 2).

For the tested phase shifter IC, the optimum states were searched based on the minimum $\mathrm{RMS}_{\varphi}$ criteria, under the conditions that $\Delta \mathrm{A} \leq 0.5 \mathrm{~dB}$ and $\mathrm{A}_{\mathrm{av}} \geq-4 \mathrm{~dB}$, with the results shown in Figure 6. Phase shifter parameters were measured for the states found; the results are shown in Table 2. For comparison purposes, the Table 2 also shows the parameters of a similar device, a serial HMC647 IC (Hittite). Therefore, using PLAN-16M to automate the measurement of a complete set of complex S-parameters, has substantially enhanced the precision parameters of the test vector phase shifter IC, bringing them to the level of its competitive analogues.

Table 2. Test results for the vector phase shifter IC.

\begin{tabular}{|l|c|c|c|c|}
\hline \multicolumn{1}{|c|}{ Object } & $\mathrm{RMS}_{\varphi}, \mathrm{deg}$. & $\mathrm{RMS}_{\mathrm{A}}, \mathrm{dB}$ & $\Delta \mathrm{A}, \mathrm{dB}$ & $\mathrm{A}_{\mathrm{av}}, \mathrm{dB}$ \\
\hline $\begin{array}{l}\text { Test phase shifter IC, states } \\
\text { according to the specification }\end{array}$ & 3.9 & 0.8 & 3.4 & -3.7 \\
\hline $\begin{array}{l}\text { Test phase shifter IC, states } \\
\text { after optimization }\end{array}$ & 0.7 & 0.14 & 0.5 & -3.9 \\
\hline HMC647, Hittite & 1.0 & - & 1.0 & -4.0 \\
\hline
\end{tabular}




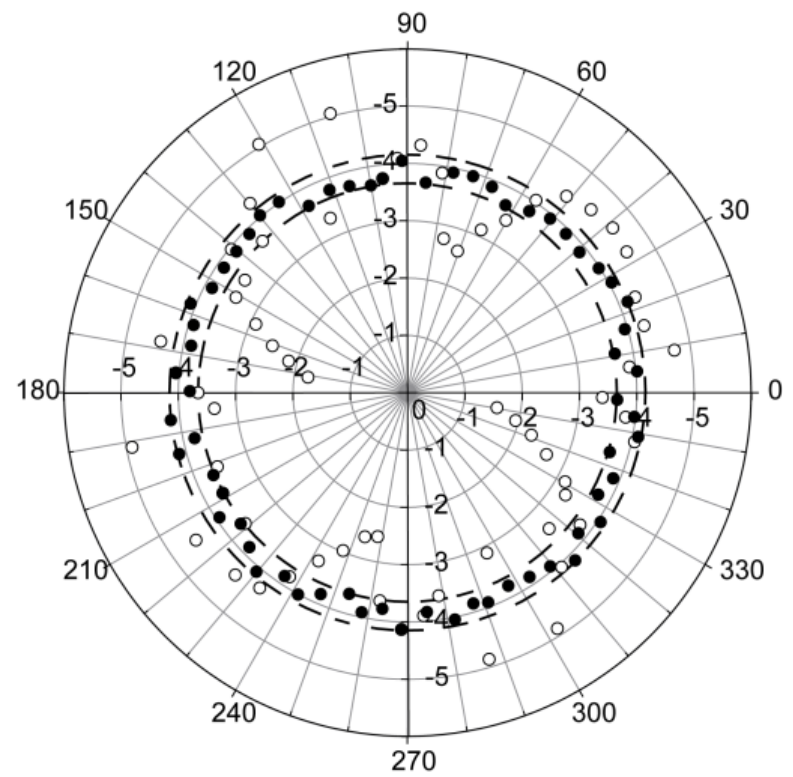

Figure 6. Amplitude-phase diagram of the vector phase shifter IC: white circles - states according to the specification, black circles - states with the minimum $\operatorname{RMS}_{\varphi}$.

\section{Conclusion}

Portable logical generator/analyzer PLAN-16M designed for automation of measurement, radiation testing and optimization of digitally controlled RF ICs, has been developed. The device is a new element for the existing RF and MW hardware-software measuring system, adapted to radiation test facility. PLAN-16M has been tested with several domestic and foreign multi-bit phase shifter and attenuator ICs. Testing of such ICs, particularly for radiation hardness, is almost non-practicable without the automation of measurement processes (involving 2048 matrices of S-parameters) and mathematical processing of the measurement results in real-time (i.e., calculating integral criteria parameters, RMS and RMSA). When used within the automated software/hardware measuring system, PLAN$16 \mathrm{M}$ provides for recording of the control word and saves the file containing measurement results in less than $1 \mathrm{sec}$. The process of measuring 2048 states of analog-digital blocks of transceiver modules for RF active phased arrays runs automatically and takes up to 100 minutes, with 70 minutes being the time needed to measure S-parameters, which depends on the measuring equipment capacity. It has been shown that the developed device can help to substantially improve precision parameters of the vector phase shifter and attenuator ICs by selecting optimal states.

\section{Acknowledgments}

The authors thank V.V. Repin and I.I. Mukhin from "Progress MRI" (Russia) for the providing the samples and their interest in the work.

This work was partially supported in accordance with agreement between Ministry of Education and Science of the Russian Federation and National Research Nuclear University MEPhI as of November 24, 2014 № 14.578.21.0075 (unique identifier of applied research: RFMEFI57814X0075). 


\section{References}

[1] J.D. Cressler, The Silicon Heterostructure Handbook: Materials, Fabrication, Devices, Circuits, and Applications of SiGe and Si Strained_Layer Epitaxy (CRC Press, London, 2005)

[2] G.N. Nazarova, V.V. Elesin, A.Yu. Nikiforov, A.G. Kuznetsov, N.A. Usachev, G.V. Chukov, CriMiCo'2014, 856 (2014) doi: 10.1109/CRMICO.2014.6959663

[3] V. V. Elesin, G. N. Nazarova, N. A. Usachev, Russian Microelectronics 39, 134 (2010) doi: 10.1134/S1063739710020083

[4] S. Shi, W. Yang, J. Zhang, Z. Chang, MATEC Web of Conferences 22, 01005 (2015) doi: 10.1051/MATECCONF/20152201005

[5] X. Liu, MATEC Web of Conferences 44, 02009 (2016) doi: 10.1051/MATECCONF/20164402009

[6] D.V. Gromov, S.A. Polevich, V.V. Elesin, CriMiCo'2009, 730 (2009)

[7] O.G. Vendik, M.D. Parnes, Antennas with electrical scanning (introduction to theory) (Science-Press, Moscow, 2002)

[8] V.V. Elesin, G.N. Nazarova, N.A. Usachev, D.I. Sotskov, G.V. Chukov, V.V. Repin, I.I. Mukhin, Izvestiya vysshikh uchednykh zavedenii. Elektronika (in Russian) 5, 31 (2012)

[9] O. A. Kalashnikov, V. V. Elesin, D. V. Gromov, CriMiCo'2014, 862 (2014) doi: 10.1109/CRMICO.2014.6959666

[10] V. V. Elesin, G. V. Chukov, D. V. Gromov, V. V. Repin, V. A. Vavilov, Russian Microelectronics 39, 122 (2010) doi: 10.1134/S1063739710020071

[11] G. V. Chukov, V. V. Elesin, D. V. Boychenko, A. G. Kuznetsov, K. M. Amburkin CriMiCo'2014, 860 (2014) doi: 10.1109/CRMICO.2014.6959665

[12] A.S. Artamonov, A.A. Sangalov, A.Y. Nikiforov, V.A. Telets, D.V. Boychenko, IEEE Radiation Effects Data Workshop 2015-January, 7004600 (2015) doi: 10.1109/REDW.2014.7004600

[13] G.G. Davydov, A.S. Kolosova, L.N. Kessarinsky, D.V. Boychenko, 2015 International Siberian Conference on Control and Communications (SIBCON), 7147094 (2014) doi: 10.1109/SIBCON.2015.7147094

[14] D.V. Gromov, A.N. Egorov, O.B. Mavritskiy, G.V. Chukov, K.M. Amburkin, CriMiCo'2015, 812 (2015)

[15] A.Y. Nikiforov, O.B. Mavritsky, A.N. Egorov, V.S. Figurov, V.A. Telets, P.K. Skorobogatov, S.A. Polevich, IEEE Radiation Effects Data Workshop, 49 (1996)

[16] Datasheet AT90USB162 IC.

URL: www.atmel.com/dyn/resources/prod_documents/doc7707.pdf

[17] Datasheet 74LVX3245 IC URL: www.fairchildsemi.com/ds/74/74LVX3245.pdf

[18] Datasheet AD826 IC URL: www.analog.com/static/importedfiles/data sheets/AD826.pdf

[19] Library of functions for MCU AT90USB162.

URL: www.atmel.com/dyn/resources/prod_documents/AVR153.zip

[20] G. V. Chukov, G. N. Nazarova, A. Yu. Nikiforov, CriMiCo'2014, 131 (2014) doi: 10.1109/CRMICO.2014.6959323

[21] Y. Zheng, C.E. Saavedra, IEEE Transactions on Circuits and Systems I: Regular Papers 57, 752 (2010) doi: 10.1109/TCSI.2009.2025851 